\title{
Analysis of the Normal-State Magnetotransport in CeIrIn
}

\author{
Sunil Nair · M. Nicklas $\cdot$ J.L. Sarrao $\cdot$ J.D. Thompson • \\ F. Steglich $\cdot$ S. Wirth
}

Received: 16 October 2008 / Accepted: 20 October 2008 / Published online: 30 October 2008

(C) The Author(s) 2008. This article is published with open access at Springerlink.com

\begin{abstract}
We present an analysis of the normal-state magnetotransport in the heavy-fermion superconductor CeIrIn 5 . The Hall effect and the transverse magnetoresistance in this material do not appear to be uniquely correlated, as inferred from the field dependence of the current ratio $\left(R_{\sigma}=\sigma_{x y} / \sigma_{x x}^{2} H\right)$. The Hall coefficient is seen to satisfy a scaling equation of the form $R_{\mathrm{H}}=f\left[H /\left(a+b T^{c}\right)\right]$. These results are compared to those observed earlier in $\mathrm{CeCoIn}_{5}$, and are discussed in terms of the contrasting phase diagram which the CeIrIn 5 system exhibits in relation to its Co counterpart.
\end{abstract}

Keywords Heavy-fermion superconductors · Hall effect . Magnetoresistance

\section{Introduction}

The phenomenon of superconductivity in the heavy-fermion metals remains an area of extensive theoretical and experimental research. The initial interest in this phenomenon was focused on understanding how a superconducting condensate could form in a regime where magnetic fluctuations are known to be dominant, since superconductivity and magnetism were thought to be antithetical to each other. This apparent contradiction is now thought to be lifted by the

S. Nair · M. Nicklas · F. Steglich · S. Wirth $(\bowtie)$

Max Planck Institute for Chemical Physics of Solids, Noethnitzer

Str. 40, 01187 Dresden, Germany

e-mail: wirth@cpfs.mpg.de

J.L. Sarrao · J.D. Thompson

Los Alamos National Laboratory, Los Alamos, NM 87545, USA fact that in these materials, the bosonic mode which facilitates Cooper pair formation may be the incipient antiferromagnetic fluctuations itself [1]. This fascinating interplay between magnetism and superconductivity has received further impetus due to the often observed existence of superconductivity in the vicinity of Quantum Critical Points (QCP) - continuous quantum phase transitions (QPT) at absolute zero temperature driven by an external control parameter. In the heavy-fermion metals, these extraordinary transitions arise as a consequence of the competition between two fundamental processes: the Ruderman-Kittel-KasuyaYosida (RKKY) interaction which promotes a magnetically ordered ground state, and the Kondo effect, which shields the local moments.

In recent years, the $\mathrm{Ce} M \operatorname{In}_{5}(M=\mathrm{Co}$, Ir or Rh) family of compounds has emerged as a fertile playground where many of these competing interactions can be individually tailored [2]. Not surprisingly, this manifests itself in the form of an extremely rich and varied phase diagram. For instance, though $\mathrm{CeCoIn}_{5}$ and $\mathrm{CeIrIn}_{5}$ are both ambient pressure superconductors, they differ drastically with respect to their quantum critical behavior in relation to the superconductivity in the magnetic field-temperature phase space. In $\mathrm{CeCoIn}_{5}$, a magnetic field-induced QCP is located in the vicinity of the superconducting upper critical field $\left(H_{\mathrm{c} 2}\right)$ [3], whereas in CeIrIn 5 , on the other hand, a QPT is speculated to be related to a metamagnetic transition that can only be approached by fields of the order of $25 \mathrm{~T}$ [4]. This marked difference is in spite of the rather similar band structures of these systems, as has been concluded from de Haasvan Alphen measurements [5, 6]. These measurements (supplemented by band structure calculations) have shown that the $\mathrm{CeM} \mathrm{In}_{5}$ systems have nearly cylindrical Fermi surfaces, which in turn arise as a consequence of their quasitwo-dimensional crystalline structures consisting of units of 
$\mathrm{CeIn}_{3}$ separated by $M \mathrm{In}_{2}$ planes. This is also manifested by a pronounced anisotropy in various physical properties. The superconductivity in some of these systems is also reported to be anomalous, and it has been suggested [7] that in $\mathrm{CeCoIn}_{5}$ a group of conduction electrons may not participate in the formation of the superconducting condensate. This can be looked upon as a rather unique form of electronic phase separation, and-if reconfirmed by other measurements-would represent the most extreme case of multiband superconductivity.

Current interest in these systems is focused on not only unraveling the rich phase diagrams which these systems exhibit, but is also stemming from the fact that many of the physical properties-both in the normal and superconducting state - are remarkably similar to those exhibited by the high-temperature superconducting cuprates [8]. For instance, in the CeMIn ${ }_{5}$ systems, the resistivity is known to have a linear temperature dependence, and the Hall coefficient is strongly temperature dependent. The superconducting gap function has line nodes and has been suggested to be of $d$-wave symmetry [9]. Moreover, the formation of the superconducting condensate appears to be preceded by a precursor state, in similarity to the pseudogap state in the cuprates. Here, we report on the analysis of the Hall effect and magnetoresistance in a single crystal of CeIrIn 5 . The correlation between the Hall and the transverse conductivities is discussed. A scaling analysis of the Hall coefficient using a functional form $R_{\mathrm{H}}=f\left[H /\left(a+b T^{c}\right)\right]$ and its implications is also reported.

\section{Experimental Techniques}

All measurements are made on a single crystal of CeIrIn ${ }_{5}$ with approximate dimensions of $0.8 \mathrm{~mm} \times 0.7 \mathrm{~mm} \times$ $0.08 \mathrm{~mm}$, using the standard six-contact geometry. Simultaneous Hall effect and magnetoresistance measurements are performed using a modified Kelvinox-25 dilution refrigerator in the range $0.05 \mathrm{~K} \leq T \leq 2.5 \mathrm{~K}$. The measurement protocol is in the form of isothermal field sweeps, with the magnetic field $H$ up to $15 \mathrm{~T}$ applied parallel to the tetragonal $c$ axis. The Hall voltages are extracted as the asymmetric component under magnetic field reversal. Low-temperature transformers are used in conjunction with low-noise voltage preamplifiers to enable a voltage resolution of better than $\pm 0.01 \mathrm{nV}$.

\section{Results and Discussions}

A comprehension of the normal-state magnetotransport of these complex systems clearly warrants the analysis of the electrical and Hall conductivities ( $\sigma_{x x}$ and $\sigma_{x y}$ respectively)

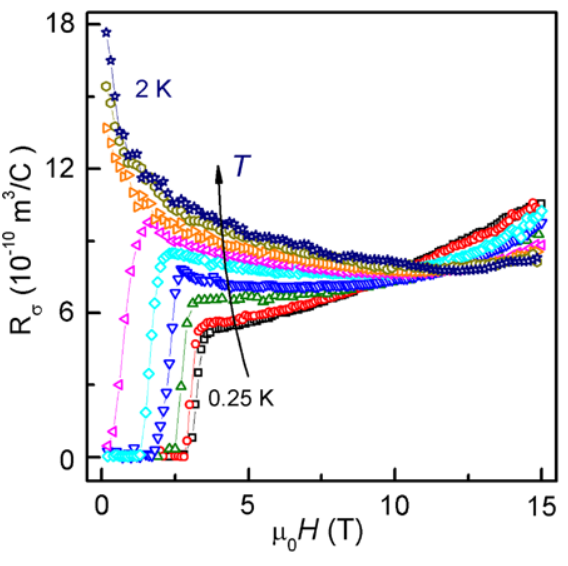

Fig. 1 Field dependence of the current ratio $\left(R_{\sigma}=\sigma_{x y} / \sigma_{x x}^{2} H\right)$ of CeIrIn $_{5}$ at selected temperatures. Unlike that reported earlier for the related system $\mathrm{CeCoIn}_{5}, R_{\sigma}$ is strongly field dependent implying that the Hall and the transverse conductivities are not uniquely correlated

in unison. An interesting manifestation of the correlation between these two quantities was recently demonstrated in $\mathrm{CeCoIn}_{5}$ : it was shown that the so-called current ratio (defined as $\left.R_{\sigma}(T, H)=\sigma_{x y} / \sigma_{x x}^{2} H\right)$ is constant with respect to the applied magnetic field below $1 \mathrm{~K}$ [10]. With this definition, the current ratio $R_{\sigma}=R_{\mathrm{H}}\left[1+\left(\tan \theta_{\mathrm{H}}\right)^{2}\right]$ differs from the conventionally used $R_{\mathrm{H}}\left(=\rho_{x y} / \mathrm{H}\right)$ at large values of the Hall angle $\theta_{\mathrm{H}}$. Here, it is to be noted that the magnitude of $\theta_{\mathrm{H}}$ which effectively measures the deflection of the charge carriers in the material due to the applied magnetic field, is substantially large in the Ce $M \mathrm{In}_{5}$ systems. In CeIrIn 5 , for instance, $\theta_{\mathrm{H}}$ attains values of the order of about $40^{\circ}$ at applied fields of the order of $15 \mathrm{~T}$ [11]. Interestingly, the constancy in $R_{\sigma}(T, H)$ was observed in $\mathrm{CeCoIn}_{5}$ in spite of the appreciable field dependence exhibited by the individual $\sigma_{x x}(H)$ and $\sigma_{x y}(H)$ components. It was suggested that this constancy of $R_{\sigma}(T, H)$ arises due to the fact that below $1 \mathrm{~K}$ the electron mean free path $(\ell)$ is significantly enhanced. Although $R_{\sigma}$ deviates from constancy above $1 \mathrm{~K}$, the application of a magnetic field helps in recovering it to an appreciable extent. This was proposed to occur because of the fact that the applied magnetic field suppresses the incipient antiferromagnetic spin fluctuations. Since spin excitations are the dominant scattering mechanism of charge carriers in this temperature range, this suppression of the spin scattering results in an enhancement of $\ell$, and consequently a correlated increase of the diagonal $\left(\sigma_{x x}\right)$ and off-diagonal conductivities $\left(\sigma_{x y}\right)$. Figure 1 shows the current ratio $R_{\sigma}$ plotted as a function of the applied field $H$. The lack of constancy, in comparison to that observed earlier in $\mathrm{CeCoIn}_{5}$, is obvious, and $R_{\sigma}$ is seen to be strongly field dependent.

The disparate behavior of $R_{\sigma}$ in the Co and Ir systems may be a reflection of the difference between the low temperature phase diagrams of these two systems: the $H-T$ phase space sampled by our measurements on $\mathrm{CeIrIn}_{5}$ does 

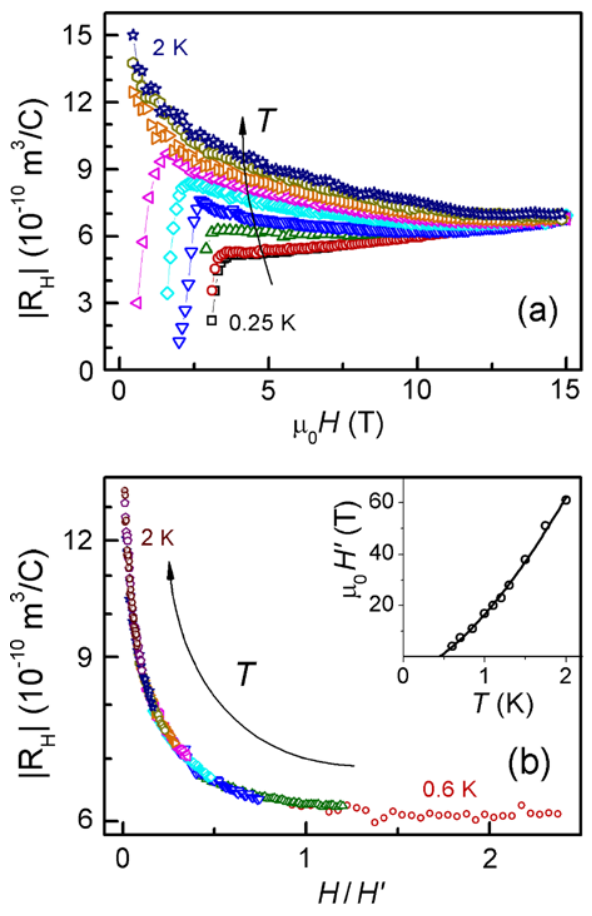

Fig. 2 The Hall coefficient $\left|R_{\mathrm{H}}\right|=\left|\rho_{x y}\right| / H$ as measured in CeIrIn at selected temperatures (a) and the scaling of the normal state $\left|R_{\mathrm{H}}\right|$ achieved by normalizing the magnetic field by $H^{\prime}(\mathbf{b})$. The inset depicts the temperature evolution of $H^{\prime}$, and the solid line is a power law fit

not encompass the putative QPT the signatures of which have been observed in prior investigations on $\mathrm{CeCoIn}_{5}$. This disparity in $R_{\sigma}$ is, however, in line with prior Hall effect measurements [12] in $\mathrm{CeCoIn}_{5}$ where a pressure dependent feature in the differential Hall coefficient was observed. This feature, attributed to the influence of critical antiferromagnetic fluctuations in $\mathrm{CeCoIn}_{5}$, was not found in $\mathrm{CeIrIn}_{5}$ [13]. More importantly, our data suggest that the field dependent excitations responsible for quasiparticle scattering in CeIrIn 5 do not appear to influence the Hall and the magnetoconductivities in a correlated fashion. This lack of correlation between the diagonal and off-diagonal magnetotransport quantities could arise due to (i) the presence of hitherto unidentified excitations which act differently on these two quantities, (ii) a non-trivial modification in the topology of the Fermi surface, or (iii) an anisotropy in the scattering rates along different areas of the Fermi surface. It is to be noted that our analysis of the Hall mobility had shown that the superconductivity in CeIrIn $n_{5}$ is preceded by a precursor state, similar to the pseudogap state in the high- $T_{\mathrm{c}}$ superconducting cuprates [13]. Using a single parameter scaling of the Hall angle, it also has recently been demonstrated that this precursor state appears to selectively influence the Hall channel, and has relatively less influence on the resistivity [11].

In the temperature regime under investigation here, the Hall coefficient $R_{\mathrm{H}}$ is in itself a quantity of fundamental in- terest. This is primarily fueled by the fact that at these low temperatures the measured Hall response in heavy-fermion systems is expected to be relatively free from the influence of skew scattering and predominantly arises only from the normal part of the Hall effect [14]. Thus, $R_{\mathrm{H}}(T, H)$ is a measure-albeit an indirect one-of the Fermi surface volume. It has been successfully used to investigate the evolution of the Fermi surface across a field-induced quantum critical point [15]. In the case of $\mathrm{CeCoIn}_{5}$, it was shown that the measured $\left|R_{\mathrm{H}}\right|$ could be scaled into a generic curve when its field dependence was normalized by a single scaling factor [12]. This factor $H^{\prime}$ (with dimension of a magnetic field) was thought to represent the effective carrier mobility $\mu_{\text {eff }}$, averaged over different sheets of the Fermi surface which contribute to the measured Hall voltage. $H^{\prime}$ was experimentally determined by the aforementioned feature in $R_{\mathrm{H}}$ in a limited temperature range, beyond which it was estimated by the scaling procedure.

Figure 2(a) depicts the $\left|R_{\mathrm{H}}\right|$ vs. $H$ as measured isothermally in CeIrIn 5 . The sharp drop in $\left|R_{\mathrm{H}}(H)\right|$ corresponds to the onset of the superconducting transition. The normalstate scaling of $\left|R_{\mathrm{H}}(H)\right|$ using a single scaling parameter $H^{\prime}$ is shown in Fig. 2(b) where a remarkably good overlap of experimental data is observed. It is to be noted that, unlike in the case of $\mathrm{CeCoIn}_{5}$, there is no discernible feature corresponding to $H^{\prime}$ in the raw $\left|R_{\mathrm{H}}(H)\right|$ data of CeIrIn 5 , and here $H^{\prime}$ is estimated in the whole temperature range using the scaling procedure alone. In line with earlier observations on $\mathrm{CeCoIn}_{5}$, the temperature dependence of $H^{\prime}$ can also be reasonably fit using a power law of the form $H^{\prime}=a+b T^{c}$ (as shown in the inset) yielding $a=-6.63 \pm 2.3 \mathrm{~T}$. In the absence of an experimental signature associated with $H^{\prime}$ in the CeIrIn ${ }_{5}$ data, the extent of uncertainty in the determination of the coefficients $b$ and $c$ (in the form of unrecognized multiplicative factors) cannot easily be ascertained. Hence, we limit our discussion to the value, and the implications of, the coefficient $a$ alone. In $\mathrm{CeCoIn}_{5}$ the value of $a$ as determined from the scaling analysis was of the order of $4 \mathrm{~T}$ and was suggested to be related to the existence of a QPT at that value of the applied magnetic field. The fact that the sign of $a$ is negative in CeIrIn 5 possibly indicates the absence of an antiferromagnetic QPT in the vicinity of superconductivity in this system. Since the magnetic field $H$ influences the effective exchange coupling between localized spins (through the RKKY interaction), the contrast in the sign of $a$ is probably related to an even weaker antiferromagnetic interaction in CeIrIn ${ }_{5}$ compared to the Co system (see discussion below). Interestingly, this scaling is observed to be valid only for data above $0.4 \mathrm{~K}$ in the case of CeIrIn 5 . We note that our measurements track only the resistive transition into superconductivity which occurs at $1.2 \mathrm{~K}$; the bulk transition in this system is known to be at about $0.4 \mathrm{~K}$. This discrepancy remains to be fully compre- 
hended though some studies have suggested that this arises due to filamentary superconductivity which is intrinsic, and involves electrons from the part of the Fermi surface responsible for bulk superconductivity [16]. The fact that this bulk transition temperature is reflected in our scaling analysisalbeit in an indirect manner-is significant. Moreover, the scaling of $\left|R_{\mathrm{H}}(H)\right|$ in CeIrIn 5 is also seen to be valid only within the coherent Kondo regime of the phase diagram. This regime is associated with a positive magnetoresistance $(M R)$, and was characterized by the change in the sign of $\partial(M R) /\left.\partial(H)\right|_{T}$ [13]. In the incoherent regime the magnetoresistance is negative, since it primarily results from the suppression of spin flip scattering. On decreasing temperature, the scattering becomes more coherent, and the magnetoresistance in this regime is driven by the Lorentz force acting on the charge carriers. The fact that this single parameter scaling is only observed in the coherent Kondo regime clearly implies that this functional form of $R_{\mathrm{H}}(T, H)$ is valid only when the contribution that arises from scattering due to spin fluctuations is absent.

A similar scaling form $R_{\mathrm{H}}=f\left[H /\left(a+b T^{c}\right)\right]$ has been reported to be valid for several members of the Ce $M \operatorname{In}_{5}$ family, albeit in a different temperature regime $\left(T>T_{\mathrm{c}}\right)$ [17]. In this (high) temperature regime, the magnitude of the scaling parameter was suggested to be related to the single-ion Kondo energy. The fact that the magnetoresistance could be used to demarcate the crossover from an incoherent to coherent Kondo scattering regime, and the observation that our scaling is valid only within the coherent Kondo regime, is in line with a two-fluid description of the Kondo lattice [18]: It was shown that while the scaling in the incoherent regime is influenced by the single-ion Kondo energy scale, the scaling in the coherent regime (as is observed in our data) should be dictated by the intersite coupling energy. The latter is a measure of the effective RKKY interaction between localized moments and is known to play a crucial role, in addition to crystal electric field splitting and the singleion Kondo scale [19]. The above-mentioned fact of an effectively weaker antiferromagnetic interaction in CeIrIn 5 in comparison to its Co counterpart is consequently manifested in the value of the intersite coupling energy scale $T^{*}(\approx 20 \mathrm{~K}$ and $\approx 45 \mathrm{~K}$ in the Ir and Co systems, respectively). The electronic ground state in these systems is clearly dictated by the strength of $T^{*}$ in relation to the single-ion Kondo scale $\left(T_{\mathrm{K}}\right)$. This is evident from the fact that $\mathrm{CeRhIn}_{5}$ (which has a magnetically ordered ground state) has a large value $(\approx 130)$ of $T^{*} / T_{\mathrm{K}}$ [20]. This ratio is found to be progressively smaller in the Co and Ir counterparts $(\approx 25$ and $\approx 7$, respectively). This qualitatively supports the results of our scaling analysis, and is also in agreement with the low-temperature phase diagrams reported for these systems.

In summary, we analyzed the normal-state magnetotransport in the heavy-fermion superconductor $\mathrm{CeIrIn}_{5}$. In the temperature regime investigated here, the Hall and transverse conductivities do not appear to be uniquely correlated. This is in marked contrast to earlier observations on CeCoIn 5 . The Hall coefficient $\left|R_{\mathrm{H}}\right|$ is seen to satisfy a single parameter scaling of the form $R_{\mathrm{H}}=f\left[H /\left(a+b T^{c}\right)\right]$ in the coherent Kondo regime. This fitting not only appears to be sensitive towards the fact that there is no QPT in the vicinity of superconductivity in this system, but also reflects (in an indirect fashion) the intrinsic nature of the disparate bulk and resistive superconducting transitions.

Acknowledgements S. Nair was supported by the Alexander von Humboldt Foundation. Work at Dresden was supported by the EC (CoMePhS 517039) and the DFG (Forschergruppe 960). Work at Los Alamos was performed under the auspices of the US Department of Energy/Office of Science.

Open Access This article is distributed under the terms of the Creative Commons Attribution Noncommercial License which permits any noncommercial use, distribution, and reproduction in any medium, provided the original author(s) and source are credited.

\section{References}

1. Mathur, N.D., Grosche, F.M., Julian, S.R., Walker, I.R., Freye, D.M., Haselwimmer, R.K.W., Lonzarich, G.G.: Nature 394, 39 (1998)

2. Sarrao, J.L., Thompson, J.D.: J. Phys. Soc. Jpn. 76, 051013 (2007)

3. Paglione, J., Tanatar, M.A., Hawthorn, D.G., Boaknin, E., Hill, R.W., Ronning, F., Sutherland, M., Taillefer, L., Petrovic, C., Canfield, P.C.: Phys. Rev. Lett. 91, 246405 (2003)

4. Capan, C., Bianchi, A., Ronning, F., Lacerda, A., Thompson, J.D., Hundley, M.F., Pagliuso, P.G., Sarrao, J.L., Movshovich, R.: Phys. Rev. B 70, 180502(R) (2004)

5. Hall, D., Palm, E.C., Murphy, T.P., Tozer, S.W., Fisk, Z., Alver, U., Goodrich, R.G., Sarrao, J.L., Pagliuso, P.G., Ebihara, T.: Phys. Rev. B 64, 212508 (2001)

6. Haga, Y., Inada, Y., Harima, H., Oikawa, K., Murakawa, M., Nakawaki, H., Tokiwa, Y., Aoki, D., Shishido, H., Ikeda, S., Watanabe, N., Onuki, Y.: Phys. Rev. B 63, 060503 (2001)

7. Tanatar, M.A., Paglione, J., Nakatsuji, S., Hawthorn, D.G., Boaknin, E., Hill, R.W., Ronning, F., Sutherland, M., Taillefer, L., Petrovic, C., Canfield, P.C., Fisk, Z.: Phys. Rev. Lett. 95, 067002 (2005)

8. Nakajima, Y., Shishido, H., Nakai, H., Shibauchi, T., Behnia, K., Izawa, K., Hedo, M., Uwatoko, Y., Matsumoto, T., Settai, R., Onuki, Y., Kontani, H., Matsuda, Y.: J. Phys. Soc. Jpn. 76, 024703 (2007)

9. Matsuda, Y., Izawa, K., Vekhter, I.: J. Phys.: Condens. Matter 18, R705 (2006)

10. Onose, Y., Ong, N.P., Petrovic, C.: Europhys. Lett. 80, 37005 (2007)

11. Nair, S., Nicklas, M., Steglich, F., Sarrao, J.L., Thompson, J.D., Schofield, A.J., Wirth, S.: arXiv:0808.2377v1

12. Singh, S., Capan, C., Nicklas, M., Rams, M., Gladun, A., Lee, H., DiTusa, J.F., Fisk, Z., Steglich, F., Wirth, S.: Phys. Rev. Lett. 98, 057001 (2007)

13. Nair, S., Wirth, S., Nicklas, M., Sarrao, J.L., Thompson, J.D., Fisk, Z., Steglich, F.: Phys. Rev. Lett. 100, 137003 (2008)

14. Fert, A., Levy, P.M.: Phys. Rev. B 36, 1907 (1987)

15. Paschen, S., Lühmann, T., Wirth, S., Gegenwart, P., Trovarelli, O., Geibel, C., Steglich, F., Coleman, P., Si, Q.: Nature 432, 881 (2004) 
16. Bianchi, A., Movshovich, R., Jaime, M., Thompson, J.D., Pagliuso, P.G., Sarrao, J.L.: Phys. Rev. B 64, 220504(R) (2001)

17. Hundley, M.F., Malinowski, A., Pagliuso, P.G., Sarrao, J.L., Thompson, J.D.: Phys. Rev. B 70, 035113 (2004)

18. Nakatsuji, S., Pines, D., Fisk, Z.: Phys. Rev. Lett. 92, 016401 (2004)
19. Aeppli, G., Yoshizawa, H., Endoh, Y., Bucher, E., Hufnagl, J., Onuki, Y., Komatsubara, T.: Phys. Rev. Lett. 57, 122 (1986)

20. Yang, Y.-f., Fisk, Z., Lee, H.-O., Thompson, J.D., Pines, D.: Nature 454, 611 (2008) 